\title{
Pseudoquiste de páncreas con extensión a mediastino*
}

\author{
Drs. ANA BELÉN ALÁEZ CH. ${ }^{1}$, CARMEN RAMIRO P. ${ }^{1}$, ALICIA CALERO A. ${ }^{1}$, \\ MARÍA DIEZ T. ${ }^{1}$, RAQUEL LATORRE F. ${ }^{1}$, RAQUEL GRAJAL M. ${ }^{1}$, \\ PURIFICACIÓN CALERO G. ${ }^{1}$, JOAQUÍN PÉREZ D. ${ }^{1}$ \\ 1 Hospital Universitario Ramón y Cajal, Madrid, España.
}

\begin{abstract}
Pancreatic pseudocyst extended to the mediastinum. Case report

We report a 53 years old male consulting for chest pain and dyspnea. On physical examination, an epigastric mass was detected. A TC scan showed a collection located in the omental bursa, which protruded over the posterior gastric wall and ascended to the mediastinum. Due to the presence of pancreatic calcifications, a pancreatic pseudocyst was suspected. The mediastinal cyst was drained percutaneously, leaving pig tail drainage in the cavity. Afterwards a cyst excision and Roux en Y gastrostomy was performed. After the surgical procedure the cyst became infected, requiring antimicrobials. After two weeks he was discharged in good conditions.
\end{abstract}

Key words: Pseudocyst, pancreatitis, mediastinum.

\section{Resumen}

Los pseudoquistes de páncreas representan el 75\% de las lesiones quísticas del páncreas y generalmente se circunscriben en el abdomen. Se presenta el caso de un paciente con un pseudoquiste de páncreas con extensión transhiatal a mediastino. Estos casos deben sospecharse mediante una historia clínica detallada y preguntando por antecedentes de dolor abdominal previo porque la clínica con la que se suelen manifestar es muy poco específica. El tratamiento de los pseudoquistes con extensión a mediastino debería ser el drenaje definitivo, bien de forma quirúrgica o endoscópica.

Palabras clave: Pseudoquiste de páncreas, mediastino.

\section{Introducción}

Los pseudoquistes de páncreas representan el 75\% de las lesiones quísticas del páncreas, generalmente estos se localizan en el interior del abdomen y se forman tras episodios de pancreatitis agudas o en pancreatitis crónicas ${ }^{1}$. La extensión y localización de los pseudoquistes a otras regiones del organismo (escroto, mediastino) son raras y se han descrito muy pocos casos en la literatura.

Se debe sospechar la extensión del pseudoquiste al mediastino en pacientes que presenten cuadro

*Recibido el 12 de junio de 2010 y aceptado para publicación el 17 de agosto de 2010.

Correspondencia: Dra. Ana Belén Aláez Ch.

Calle Gijón, 16. Puertollano. Ciudad Real, España.

anabelenalaez@hotmail.com 


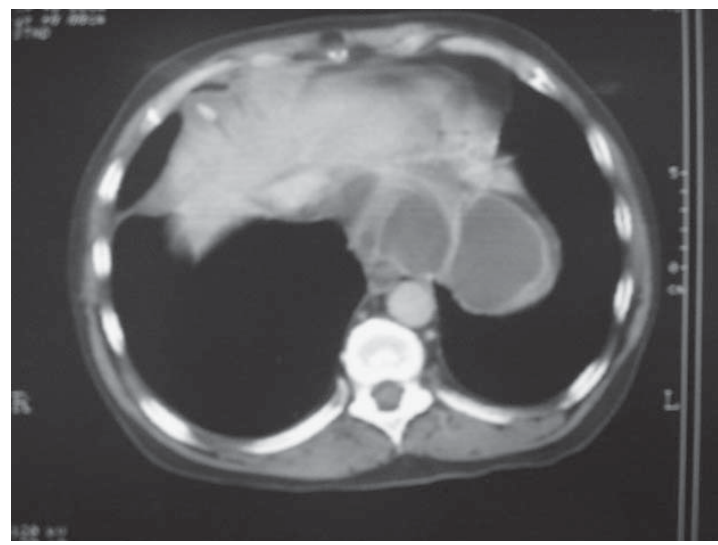

Figura 1. TAC: colección.

de disnea, dolor torácico o incluso disfagia. Para su diagnóstico es importante la sospecha clínica ya que, si no se pregunta por episodios previos de dolor abdominal y no se relaciona esta clínica con la pancreatitis del paciente, el cuadro puede ser diagnosticado de forma tardía, lo que conllevaría a un empeoramiento del pronóstico del paciente.

\section{Caso clínico}

Varón de 53 años de edad que acude al servicio de urgencias por dolor torácico y disnea de moderados esfuerzos de varios días de evolución. No refiere fiebre ni otra sintomatología en el momento de su llegada a urgencias.

A la exploración física se encuentra consciente y orientado, saturación $\mathrm{O}_{2}$ del 85\%, estable hemodinámicamente, frecuencia cardíaca de 95 y frecuencia respiratoria de 20. El abdomen es blando, depresible, no doloroso a la palpación y en epigastrio se palpa una dudosa masa que protruye y que parece depender de planos profundos sin aumento del dolor a dicho nivel. No presenta signos de irritación peritoneal.

En la analítica destaca una elevación de transaminasas (ya existente en analíticas previas) y leucocitosis de 14.500 sin neutrofilia. Se realiza radiografía de tórax, donde se objetiva una ocupación parcial de hemitórax derecho y derrame pleural. Se decide realizar una punción del derrame con análisis bioquímico del mismo y en el resultado se observa una amilasa de 3.500. Ante estos hallazgos se decide realizar una TAC tóraco-abdómino-pélvico (Figura 1 ), en la que se observa una colección en la transcavidad de los epiplones que protruye sobre la cara posterior del estómago y que asciende a mediastino, sobre todo en el lado derecho. Se observa, a su vez,

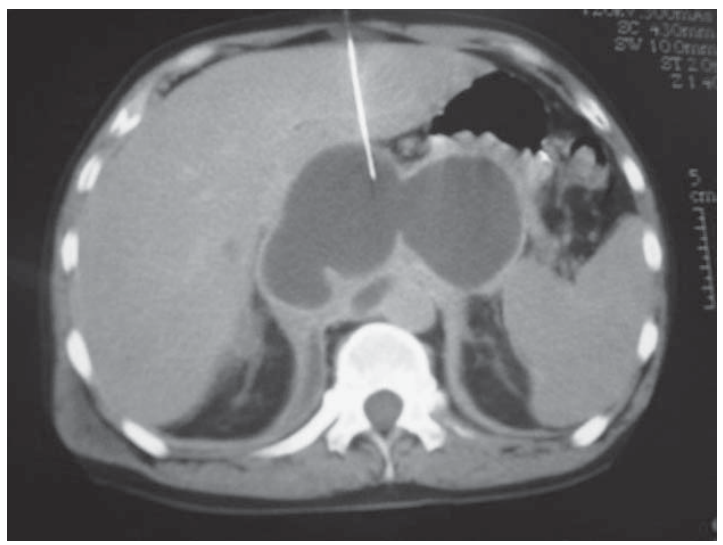

Figura 2. Punción bajo TAC.

alguna calcificación pancreática que hace sospechar el diagnóstico de pancreatitis crónica.

Reinterrogando al paciente, este nos cuenta que ha presentado episodios ocasionales y autolimitados de dolor en epigastrio en los últimos meses y se evidencia un hábito enólico, por lo que es diagnosticado de pancreatitis crónica de origen enólico y sospecha de pseudoquiste asociado con extensión al mediastino.

El tratamiento recibido consiste, en primer lugar, en la punción del pseudoquiste a nivel del mediastino mediante la colocación de un pig-tail (Figura 2) y, una vez que la colección ha disminuido de tamaño y tras la resolución de los síntomas, se decide intervención quirúrgica programada realizando una quistogastrostomía en Y de Roux. Tras la intervención el paciente evoluciona favorablemente, aunque permanece ingresado durante dos semanas por sobreinfección del pseudoquiste que precisa antibioterapia. Es dado de alta asintomático y con TAC de control en el que no se observa el pseudoquiste abdominal ni mediastínico.

\section{Discusión}

Los pseudoquistes de páncreas deben sospecharse por la clínica y deben ser confirmados mediante pruebas de imagen (TAC abdominal, ecografía endoscópica, RMN). Según la clasificación de Atlanta ${ }^{2}$, se define un pseudoquiste de páncreas como una acumulación de tejidos, detritus, enzimas pancreáticos y sangre que carece de revestimiento epitelial y cuya pared está compuesta de tejido necrótico, tejido de granulación y tejido fibroso. Para poder hablar de pseudoquiste pancreático deben pasar al menos 6 semanas desde el episodio de pancreatitis, ya que antes de este tiempo lo que se produce generalmente 
son colecciones agudas de líquido pancreático que pueden tener distinta evolución (resolverse en el tiempo, abscedarse o transformarse en pseuquistes).

Según datos de la literatura, los pseudoquistes se pueden complicar en torno a un $10 \%-25 \%$, siendo más frecuente su complicación en pancreatitis crónicas que en agudas ${ }^{3}$.

Se piensa que los pseudoquistes mediastínicos se producen por la ruptura de los conductos pancreáticos al espacio retroperitoneal y el líquido pancreático asciende al mediastino a través del hiato diafragmático aórtico o esofágico. Hasta ahora se han descrito unos 50 casos en la literatura ${ }^{4}$.

Los síntomas se producen generalmente por la ocupación y compresión mediastínica que provoca y suelen ser disfagia, pseudoacalasia, dolor torácico, disnea. El diagnóstico debe sospecharse en pacientes que presenten estos síntomas y tengan antecedentes recientes de pancreatitis agudas o crónicas.

La primera prueba de imagen que suele realizarse es una radiografía de tórax; sin embargo, esta suele ser inespecífica ya que sólo suele verse una opacidad retrocardial o derrame pleural. Aun así, la prueba que nos dará el diagnóstico casi de certeza es una TAC tóraco-abdominal que tiene una sensibilidad de un 90\%-95\%, y además aporta información acerca de la localización del pseudoquiste y de su relación con los órganos vecinos. La ecografía abdominal tiene menor sensibilidad (75\%). Sin embargo, la ecoendoscopia es un elemento diagnóstico cada vez más utilizado, ya que no sólo aumenta de forma importante la sensibilidad de la ecografía abdominal sino que además permite la punción del quiste/ pseudoquiste de páncreas para afinar más en el diagnóstico.

Los síntomas del paciente vendrán derivados de la complicación y extensión del pseudoquiste; así, éste puede sobreinfectarse, sangrar o aumentar de tamaño hasta extenderse a mediastino, ingle o escroto y producir sintomatología relacionada con la zona a la que se han extendido. En nuestro caso, la clínica por la que el paciente consultó se debió a la extensión torácica y a la compresión de estructuras mediastínicas (disnea, dolor torácico...).

La resolución de los pseudoquistes de páncreas según lo publicado en la literatura varía entre un 8 y un $80 \%^{6}$. Esta variabilidad se debe probablemente a la gran variabilidad en cuanto al tamaño de los pseudoquistes en las distintas series en la literatura. Algunos factores que disminuyen la posibilidad de resolución espontánea del pseudoquiste son: tamaño mayor de $10 \mathrm{~cm}^{7}$, localización en cola pancreática $^{8}$, pseudoquiste con paredes gruesas $^{9}$, asociación con estenosis del conducto pancreático ${ }^{9}$ y desarrollo de pseudoquistes extrapancreáticos en pancreatitis alcohólicas ${ }^{10}$. Aun así, la resolución espontánea de los pseudoquistes mediastínicos es rara, y debido a su escasa frecuencia no se han podido establecer unas pautas y condicionantes de tratamiento conservador o quirúrgico. En algunos casos se ha observado la resolución espontánea de algún pseudoquiste con extensión mediastínica con uso de octreótide ${ }^{11}$.

El tratamiento no quirúrgico incluye el drenaje percutáneo guiado por ecografía o por TAC, que presenta riesgo de sobreinfección del pseudoquiste y la posibilidad de establecer una fístula pancreática, o bien el drenaje endoscópico, el cual sólo es técnicamente posible en caso de compresión e impronta gástrica o duodenal que sea visible en endoscopia gastroduodenal o en ecoendoscopia. Esta técnica está en auge en algunos centros y parece tener buenos resultados en manos expertas (en algunas series se dan tasas de resolución del $85 \%{ }^{12}$ ). El drenaje transpapilar mediante colangiografía endoscópica se ha usado también en el tratamiento de estos pseudoquistes, aunque para poder realizarlo es necesario que exista una comunicación entre el pseudoquiste y el conducto pancreático.

En cuanto al tratamiento quirúrgico existen dos posibilidades: el drenaje interno y el externo. El drenaje interno, o derivaciones quistodigestivas, están indicadas en los pseudoquistes sintomáticos en los que la pared sea lo suficientemente consistente y sólida para poder ser suturada. Esta derivación se puede realizar a estómago (quistogastrostomía), a duodeno (quistodudodenostomía) o a un asa de yeyuno (quistoyeyunostomía en Y de Roux). En el caso del externo, se deja un drenaje en la cavidad quística y está indicado, al igual que el drenaje percutáneo, en pseudoquistes infectados.

En nuestro paciente se optó por la derivación quirúrgica porque a pesar de que se colocó un pig tail en el pseudoquiste este no se resolvió de forma adecuada ni definitiva. No se realizó esta técnica por vía laparoscópica por no tener manejo en este tipo de patologías, aunque actualmente estaría indicada la vía laparoscópica en grupos con experiencia en dicha técnica.

Como conclusión podríamos decir que los pseudoquistes con extensión mediastínica son raros y que, dada su clínica inespecífica, es imprescindible su sospecha preguntando por episodios previos de dolor abdominal y por el posible hábito enólico del paciente. Su diagnóstico definitivo se realiza con TAC o ecoendoscopia con punción del líquido y demostrando elevación de amilasa y ausencia de marcadores tumorales, y su tratamiento hoy en día es controvertido debido a su escasa frecuencia aunque deberían considerarse como pseudoquistes complicados e intervenirlos bien endoscópicamente o bien mediante cirugía. 


\section{Referencias}

1. Fernández JA. Tumores quísticos del páncreas: revisión de la literatura. Cir Esp. 2003;73:297-308.

2. Bradley EL $3^{\text {rd }}$. A clinically based classification system for acute pancreatitis. Arch Surg. 1993;128: 586-90.

3. Vosoghi M, Sial S, Garrett B, Feng J, Lee T, Stabile BE, Eysselein VE. Medscape Gen Med. 4. Posted on July 18, 2002.

4. Rose EA, Haider M, Yang SK. Mediastinal extension of pancreatic pseudocyst. Am J Gastroenterol. 2000;95:3638-9.

5. Frenzer A, Schubart P, Soucek M. Disappearance of a large mediastinal pseudocyst in a patient with chronic alcoholic pancreatitis after total parenteral nutrition. Eur J Gastroenterol Hepatol. 1995;7:369-71.

6. Mehta R, Suvarna D, Sadasivan S, John A, Raj V, Nair $P$, et al. Natural course of asymptomatic pancreatic pseudocyst: A prospective study. Indian J Gastroenterol. 2004;23:140-2.
7. Aranha GV, Prinz RA, Esguerra AC, Creenlee HB. The nature and course of cystic pancreatic lesions diagnosed by ultrasound. Arch Surg. 1983;118:486-8.

8. Maringhini A, Uomo G, Patti R, Rabitti P, Termini A, Cavallera A, et al. Pseudocysts in acute nonalcoholic pancreatitis: incidence and natural history. Dig Dis Sci. 1999; 44:1669-73.

9. Forsmark CE, Grendell J. Complications of pancreatitis. Semin Gastrointets Dis. 1991;2:165-76.

10. Gouyon B, Levy P, Ruszniewski P, Zins M, Hammel P, Vilgrain V, et al. Predictive factors in the outcome of pseudocysts complicating alcoholic chronic pancreatitis. Gut 1997;41:821-5.

11. Yasuda H, Ino Y, Igarashi H, Arita Y, Nakamuta M, Sumii T, et al. A case of pancreatic pleural effusion and mediastinal pancreatic pseudocyst: Management by a somatostatin analogue octreotide. Pancreas 1999;19:410-2.

12. Vidyarthi G, Steinberg SE. Endoscopic management of pancreatic pseudocyst. Surg Clin N Am. 2001;81:40510. 